\title{
Radiology and Physics. ${ }^{1}$
}

\author{
By Dr. G. W. C. KaYe.
}

$\mathrm{T}$ HE appreciation of the physicist by the medical worker in this country is of recent growth, but radiologists, while fully alive to the enormous part that radiology will play in medicine in the future, are only awakening to the fact that, if radiology is to advance as it should, they will have to correlate it continuously with physics. They may not find such correlation very easy. Not that physicists would look askance at the idea ; the difficulty is that there are so few of them who are interested. The physicist has never been taught to look upon radiology as offering a possible career. Even had he been prepared to risk it, he would not have found educational facilities to put him on his way. There are probably not half a dozen physicists employed in radiology in this country. The Germans discovered, long before the war, that the secret of progress in radiology was to bring the medical man and physicist continually together and let them work side by side. They went further and introduced them both to the manufacturer-but that is another story! Is the British radiologist in a position to submit techniques, backed up with a wealth of physical and scientific data such as the German has recently given to the world? It is to be hoped so, but the British radiologist is sadly handicapped by not being able to look to the physicist ultra-violet ray and the longest $\mathrm{X}$-ray, but within the last few months it has been discovered that the continuity is complete and that the X-rays follow on and, indeed, overlap the ultra-violet end of the spectrum. The study of this missing group of octaves had invited attention for some time. The grating method proved unavailing for the purpose, the wave-lengths being too small for our artificially ruled gratings and too big for crystal gratings. Further, at either end of the gap the vacuum spectrometer had proved necessary owing to the extremely absorbable nature of the rays. The problem has finally been attacked with success in this country and America by Millikan, Richardson, Hughes, and Kurth who, using indirect photoelectric methods, have traced X-ray spectrum lines of various elements right across the gap and into the already explored ultra-violet. Fig. I shows the positions of some of these lines.

The following are the wave-lengths in Angström units, i.e. $\mathrm{Io}^{-8} \mathrm{~cm}$. of the regions of the spectra we have been discussing :-

$\begin{array}{ll}\text { Visible light } & 7200 \text { to } 4000 \\ \text { Ultra-violet light } & 4000 \text { to } 200 \\ \text { X-rays } & 500 \text { to } 0 \cdot 06 \\ \gamma \text {-rays } & 1 \cdot 4 \text { to } 0 \cdot 01\end{array}$

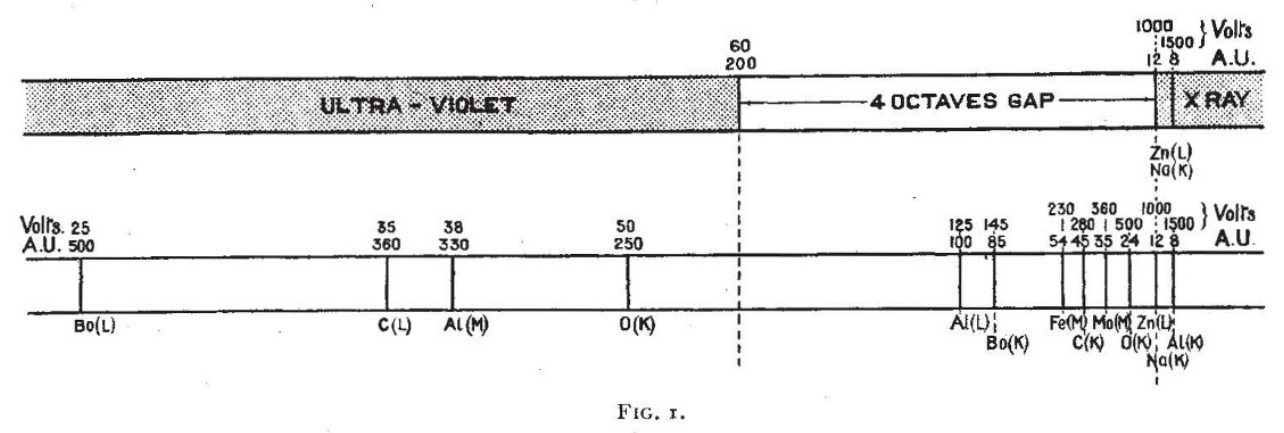

for the discharge of duties which he has neither time nor, possibly, inclination to see to himself.

Radiology needs men who have a sound knowledge of the physics of radiology and are, furthermore, well grounded in electrical engineering, especially on the high-tension side of the subject. If we could ensure a steady supply of qualified physicists and electrotechnicians who knew that in their future work they need not fear that they will not enjoy, both professionally and socially, the full status of their medical colleagues, we could look forward to a desirable allround improvement in the science and art of British radiology.

Within the last decade we have learnt that X-rays are identical with light rays in almost every particular, the main difference being that the wave-lengths of the $\mathrm{X}$-rays are much shorter. Until recently, a gap of about 4 octaves existed between the shortest known

\footnotetext{
1 Abridged from the Mackenzie-Davidson Memorial Lecture delivered on February 17 at the Royal Society of Medicine.
}

It thus appears that we can now claim a knowledge of the existence of over ${ }_{3} 3$ octaves of X-rays or, including radium $\gamma$-rays, nearly $I 6$ octaves. As yet the radiologist has only turned about 3 octaves of these to account.

As is now well known, the parallelism between light rays and $\mathrm{X}$-rays is maintained by the presence of spectrum lines in the X-ray spectra. Just as the spectrum of a hot body normally consists of a continuous spectrum of white light, together with certain spectrum lines the wave-lengths of which are characteristic of the radiating material, so an element emitting $\mathrm{X}$-rays not only gives out " white" radiation, but superposes its characteristic lines on the general spectrum. The characteristic X-ray spectra are found to be much less complicated than light spectra and are more readily sorted out into groups or series of associated lines. These several series, each of which includes a number of lines, are designated-J, K, L, M-and are broadly differentiated by a progressive increase in the average 
wave-length of each group as we pass from one to another, series $\mathrm{J}$ having the shortest wave-length and requiring the highest voltage to excite it. It should be added that all the constituent lines of a group are excited simultaneously at a critical minimum voltage.

The work on X-ray spectra has thrown great light on the structure of the atom, and, in passing, it may be recalled that present-day theory regards all atoms, of whatever kind, as built up of two kinds of "bricks," and two only-(a) negatively charged electrons, and $(b)$ hydrogen " nuclei," each more than I 800 times as heavy as an electron and carrying a charge equal to that on the electron, but positive in sign. Rutherford's nucleus theory of the atom, now universally accepted, regards an atom as built up of a minute positive nucleus (to which practically the whole mass of the atom is attributed) surrounded by a cluster of electrons grouped in rings. The total number of electrons in these rings is equal to the atomic number $(\mathrm{N})$ of the atom in question. The nucleus of the atom is regarded as built up of hydrogen nuclei held together by electrons, the former being in excess to just such an extent that the nucleus as a whole contains $\mathrm{N}$ positive charges. This serves to counterbalance the $\mathrm{N}$ negative charges of the clectron rings, the result being an electrically neutral atom. For example, platinum has an atomic number of 78 . Its atomic weight determined chemically is 195 . Thus, if platinum is a simple element, the platinum atom has a nucleus composed of r95 hydrogen nuclei and II 7 electrons, the difference $(78)$ serving to counterbalance the 78 electrons in the rings. The various elements differ only one from another in that they have different nuclear charges, the nucleus determining the mass and radioactive properties, while the number and grouping of the cluster of electrons in the rings control the chemical and spectroscopic properties. For example, the $\mathrm{K}$ radiation is supposed to arise from the displacement of an electron in the innermost ring, the $\mathrm{L}$ radiation from the next ring, and so on.

Within the last few years it has been established experimentally that there is a definite boundary to every spectrum of general X-rays on its short wave side. The position of this boundary (or quantum limit) is not affected by the nature of the element emitting the $\mathrm{X}$-rays, but is dependent solely on the maximum voltage applied to the tube. The relationship is given by the well-known quantum equation of Planck. Substituting the accepted values of the constants, it follows that

$$
\text { max. voltage }=\frac{12,400}{\text { shortest wave-length in A.U. }}
$$

This very simple relation provides us with a scale of quality which, if not perfect, is more exact than any which the radiologist has been in the habit of using. Spectral curves of X-ray intensity are not symmetrical, the shortest waves are the dominating ones. The mean effective wave-length (or "centre of gravity") of a spectrum of rays approximates to the wave-length of the peak of the curve, i.e. the wave-length of maximum intensity. Now there is some evidence that this wave-length of the peak $\left(\lambda_{m}\right)$ is proportional to the limiting or quantum wave-length $\left(\lambda_{0}\right)$; in many cases $\lambda_{m}$ proves to be approximately $4 / 3$ times $\lambda_{o}$. But in practice it is much easier to measure $\lambda_{\theta}$ than $\lambda_{m}$, and this fact gives an added importance to the measurement of the quantum limit and enables us to identify very fairly the quality of a mixed bundle of X-rays. No doubt something depends on the wave-form of the exciting potential, but the effect of this is probably less important as the voltage is raised. The precision of the method would be enhanced if steps were taken to standardise apparatus and technique, so that all work could be done by the use of, at most, three or four spectra the distinctive features of which, including energy distribution, could be determined and specified.

But how is the radiologist going to measure wavelengths in his operating room? At present, the easiest plan appears to be by measuring the maximum voltage and using Planck's relation. The voltage can be obtained by use of a reliable type of electrostatic voltmeter, or, failing that, by measuring the alternative gap by means of some approved type of spark gap such as the sphere gap. Another and a better plan is to measure the quantum limit by means of a portable direct-reading spectrograph of the type designed in Germany by Seemann (Fig. 2). Incidentally, these direct-reading spectrographs act as very convenient

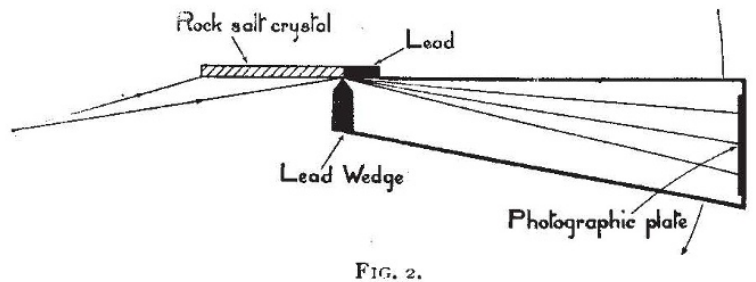

and accurate high-tension voltmeters, which afford a measure of the true maximum voltage effectively operating a tube.

There are two things that may happen to a beam of $\mathrm{X}$-rays when passing through a material. Part of it may be absorbed, and is therefore wholly transformed into characteristic radiations of the material, the process always being accompanied by the liberation of electrons. The rest of the beam is scattered or dispersed which, in effect, is equivalent to stating that while the rays are unaltered in quality a considerable proportion of them have their direction altered. Scattering, which finds a close parallel in the dispersion of light by a fog, is more noticeable with light atoms than with heavy.

We explain these two effects-absorption and scattering-by supposing that absorption is caused by the flicking off by the X-ray of an electron in one of the ring systems in the atom. The outcome is the vibration of the ring systems in question with characteristic periods and the expulsion of an electron from the atom at high speed. If, on the other hand, it happens that the X-ray is incapable of definitely ejecting an encountered electron but merely jars it, so to speak, then the electron, having absorbed the energy of the X-ray, vibrates not with its own free period but with a forced period which is prescribed by the X-ray and it re-emits its new-found energy in all directions, though chiefly round and about the original direction. With a medium weight or heavy atom the proportion of scattered to absorbed radiation depends upon the wave-length and 
may be small. With a light atom the amount of scattered radiation is almost always large.

The problems of scattering have come to the fore recently in radiology in connection with deep therapy. The human body is made up chiefly of carbon, hydrogen, and oxygen - all light atoms - and its ability to scatter $\mathrm{X}$-rays in the adjacent air has long been familiar to radiologists, especially in screening work. But the extent of the scattering within the body itself is just as marked, and this is the case whether the rays are of medium or high penetration. It has been established by Dessauer and others, from measurements made on specified areas at various depths within the tissue, that from 60 to 80 per cent. of the effectiveness of highly penetrating rays is due to scattered rays which originally were not directed at the area in question.

The subject of protection has recently excited a great deal of attention by reason of a series of casualties to prominent radiologists. The various radiological societies and institutions in London co-operated in I $92 \mathrm{I}$ in the formation of a representative Committee, which was asked to go into the whole question and draw up recommendations for the guidance of all concerned. It was agreed that the question of the protection of the operator was the sole issue ; the existing measures had proved to be adequate so far as the patient was concerned. The word "protection " was to be interpreted in a wide sense.

The Protection Committee was fortunate in securing Sir Humphry Rolleston as Chairman and, under his eminent leadership, has already drawn up two memoranda. They need not be referred to in detail here, but they have already been widely acted upon, and there is little doubt that presently the bogey of X-ray dangers will have been laid. In years to come this country will be entitled to congratulate itself on having given a lead to the world in this matter.

The Committee has laid down certain standards of protection against $\mathrm{X}$ - and $\gamma$-rays, which are expressed very simply in terms of the equivalent thickness of sheet lead. These thicknesses were based on available experimental data; for example, the X-rays from a tube excited by about 180,000 volts are cut down over ro, 000 times by $3 \mathrm{~mm}$. of lead, and over I million times by ro $\mathrm{mm}$. of lead. The choice of the actual protective material may, of course, be determined by insulating, electrostatic, or other considerations, but its thickness should be such as to provide protection equivalent to the amount of lead specified.

The Committee sought and secured the co-operation of the National Physical Laboratory, both in investigatory work and in the question of the inspection of existing X-ray departments in hospitals and other institutions. The N.P.L. has already inspected a number of X-ray departments throughout the country, and it may be said at once that, if the conditions which obtained there may be regarded as typical, the Protection Committee needs no justification in its labours. The Committee has adopted the common-sense principle that, wherever possible, the tube box or enclosure should form a complete shield in all directions, allowing only the minimum aperture for the work in hand. Few installations subscribe to this very reasonable demand.
In some cases the scattered radiation in different parts of the X-ray rooms proved to be so excessive as to prohibit examination by electroscope, and observations had to be confined to noting the comparative ease with which the bones of the hand could be seen on a screen as it was carried round the room.

It is established that ventilation is of prime importance but, unfortunately, the radiological departments are generally situated in the basement. Ventilation difficulties are multiplied tenfold in consequence and, further, the rooms are largely shut off from the beneficent effects of sunshine. In the majority of cases the high-tension system consists of stretched over-head small gauge wires, connected by spring tapes, or spiraliy wound fine wires to the various apparatus. The resulting brush discharge produces ozone in abundance, and, as extractor fans are rarely fitted, the unfortunate operator gets the full benefit. The Protection Committee has recommended the use of smooth tubes or rods or heavily insulated wires with the object of abolishing the evils of brush discharge. It suggests the employment of commodious rooms with ample head room, especially in the case of deep therapy outfits where the exciting voltages are in the region of 200,000 . Another danger is here indicated; more than one fatality has been occasioned by accidental discharge to an operator working in a small room with slack or looped high-tension wires.

Generous recognition should be paid to the X-ray manufacturers of this country for the way they are beginning to co-operate with the Committee. The British X-ray manufacturers, divided as they are, are mostly carrying on under great difficulties at the present time. Yet, despite their difficulties, almost all of them have taken steps to obtain from the National Physical Laboratory test figures for the various protective materials which they are incorporating into existing and new installations. Such measurements are rapidly and inexpensively carried out by the Laboratory, and no radiologist need deny himself the security which the N.P.L. certificate affords. The Laboratory experience amply confirms the necessity for such tests. For example, lead glass has been tested -of which only $5 \mathrm{~mm}$. were required to give the protection of I mm. of lead. For other samples of glass as much as ro mm. were required. The corresponding figures for lead rubber show variations between $I \cdot 7$ $\mathrm{mm}$. and $4 \mathrm{~mm}$. as the equivalent of $\mathrm{I} \mathrm{mm}$. of lead. Thus, with either protective material a manufacturer can easily be 100 per cent. out in his reckoning if he employs uncertified material. He owes it to himself and his customers to take no such risks.

Two noteworthy steps, pregnant with promise for the future of radiology, were taken in the establishment of the Diploma in Radiology and the formation of the Society of Radiographers. Here again this country has taken the lead. And when some day we get an Institute of Radiology, with which Mackenzie-Davidson's name should be associated in some way, a further great step will have been taken to assist radiology in this country to take the proud position among the sciences to which its important and beneficent activities entitle it. 